\title{
Combined fetal neural transplantation and nerve growth factor infusion: effects on neurological outcome following fluid-percussion brain injury in the rat
}

\section{Grant Sinson, M.D., Madhu Voddi, B.A., and Tracy K. McIntosh, Ph.D.}

Division of Neurosurgery, University of Pennsylvania, School of Medicine, Philadelphia, Pennsylvania

This study was designed to evaluate the histological and behavioral impact of fetal neural transplantation with and without neurotrophin infusion in rats subjected to traumatic brain injury using a clinically relevant model of lateral fluid-percussion brain injury. Adult male Sprague-Dawley rats received lateral fluid-percussion brain injury of moderate severity (2.1-2.3 atm). Twenty-four hours after injury, minced fetal cortical grafts (E16) were stereotactically transplanted into the site of injury cavity formation (in 32 rats). Ten control animals received injections of saline. A third group of 29 animals that received transplants also underwent placement of a miniosmotic pump (immediately after transplantation) to continuously infuse nerve growth factor (NGF) directly into the region of graft placement for the duration of the experiment. A fourth group of eight animals underwent transplantation of fetal cortical cells that had been dissociated and placed in suspension. Animals were evaluated at 72 hours, 1 week, and 2 weeks after injury for cognitive function (using the Morris water maze), posttraumatic motor dysfunction, and transplant survival and morphology (using Nissl and modified Palmgren's silver staining techniques). Robust survival of whole-tissue transplants was seen in $65.6 \%$ of animals and was not increased in animals receiving NGF infusion. Animals receiving transplants of cell suspension had no surviving grafts. Brain-injured animals receiving transplants showed significant cognitive improvements compared with controls at the 2-week evaluation. Significantly improved memory scores were seen at all evaluation times in animals receiving both NGF and transplants compared with injured controls and compared with animals receiving transplants alone at the 72-hour and 1-week evaluations. Neurological motor function scores were significantly improved in animals receiving transplants alone and those receiving transplants with NGF infusion. Histological evaluation demonstrated differentiation of grafted cells, decreased glial scarring around transplants when compared with control animals, and the presence of neuronal fibers bridging the interface between graft and host. This study demonstrates that fetal cortical cells transplanted into the injured cortex of the adult rat can improve both posttraumatic cognitive and motor function and interact with the injured host brain.

Key Words * fluid-percussion injury * nerve growth factor * neural transplantation * cognitive dysfunction * traumatic brain injury 
For many years neural transplantation has been studied as a possible means of relieving central nervous system (CNS) dysfunction. Initial studies examined the long-term viability of transplanted cells and the feasibility of developing functionally significant graft-host interactions. Transplantation techniques have also proven to be a powerful tool in neuroscience research, leading to the demonstration that transplant-derived soluble products could improve neurological function in injured rats.[6,24,32] The capacity for adult CNS neurons to regrow after axotomy was also established using experimental transplantation paradigms.[1]

According to a report on patients with Parkinson's disease,[28] transplantation of adrenal medullary cells into the caudate nucleus to provide additional dopamine has resulted in encouraging preliminary results. This report reemphasized the potential utility of transplantation as a neuroprotective strategy, in addition to its role as a research tool. Technical refinements have resulted in the use of fetal neural grafts rather than adrenal medullary cells in patients with Parkinson's disease. These grafts send out processes and make dopamine after implantation.[59] Additional clinical studies using these techniques have reported positive results and are continuing to accrue patients. $[9,10,52]$ In models of traumatic spinal cord injury, transplanted neural tissue has been shown to survive, bridge gaps in the injured spinal cord, decrease glial scarring, and even make functional connections with the host.[2,21,23,37,38]

The use of various growth factors to modify the microenvironment after transplantation has been shown to improve graft survival and function.[13,30,41,56] After experimental brain injury in rats, nerve growth factor (NGF) receptors have been shown to decrease,[27] whereas NGF infusions have recently been shown to improve cognitive outcome.[43] The histological correlates and cellular mechanisms that underlie these improvements have yet to be identified.

The lateral fluid-percussion model of brain injury in the rat results in well-characterized outcomes that reproduce many components of human head injury.[11,29,36] Previous studies have demonstrated that fetal neural transplants survive when grafted in the acute posttraumatic period in this injury model.[48] In the present study we hypothesized that fetal neural grafts transplanted into rats that had undergone fluid-percussion brain injury might result in an attenuation of cognitive and neuromotor deficits. The possibility that NGF infusion might improve the graft survival and further impact behavioral outcomes was also evaluated.

\section{MATERIALS AND METHODS}

\section{Surgical Procedures}

Experimental Paradigm. Seventy-nine male Sprague-Dawley rats, each weighing between 350 and 400 $\mathrm{g}$, were anesthetized with $60 \mathrm{mg} / \mathrm{kg}$ sodium pentobarbital administered intraperitoneally. The lateral (parasagittal) fluid-percussion model of brain injury used in this study has been described previously.[29] Briefly, a 5-mm craniectomy was performed over the left parietal cortex midway between the lambda and bregma. The injury was delivered after a hollow Leur-loc fitting was cemented to the craniectomy site and the Leur-loc then attached to the fluid-percussion device. All animals received brain injury of moderate severity (2.1-2.3 atm). After injury, the Leur-loc construct was removed and the skin was sutured. Normothermia was maintained with warming pads until the animals began to walk. Brain temperature was not directly monitored because it has previously been shown that use of warming pads maintains normal brain temperature in this model of brain injury.[34]

After brain injury, all animals were separated into one of four groups: Group 1 (32 rats) received 
transplants of minced fetal brain tissue (E16) 24 hours postinjury directly into the site of maximal cortical injury; Group 2 (10 rats) received an injection of saline alone into the site of maximal injury 24 hours after injury; Group 3 (29 rats) received a combination of fetal cell transplants and continuous infusion of NGF beginning 24 hours postinjury; and Group 4 (eight rats) received transplantation of dissociated fetal neuron suspension in the site of maximal injury 24 hours postinjury.

Transplantation Studies. Animals in Group 1 were reanesthetized with $60 \mathrm{mg} / \mathrm{kg}$ sodium pentobarbital injected intraperitoneally 24 hours after fluid-percussion brain injury, and a 1-mm burr hole was made 2 $\mathrm{mm}$ lateral to the craniectomy site (Fig. 1 upper). This corresponds to the area of maximal cortical injury seen in this model (Fig. 1 lower).[5,48]

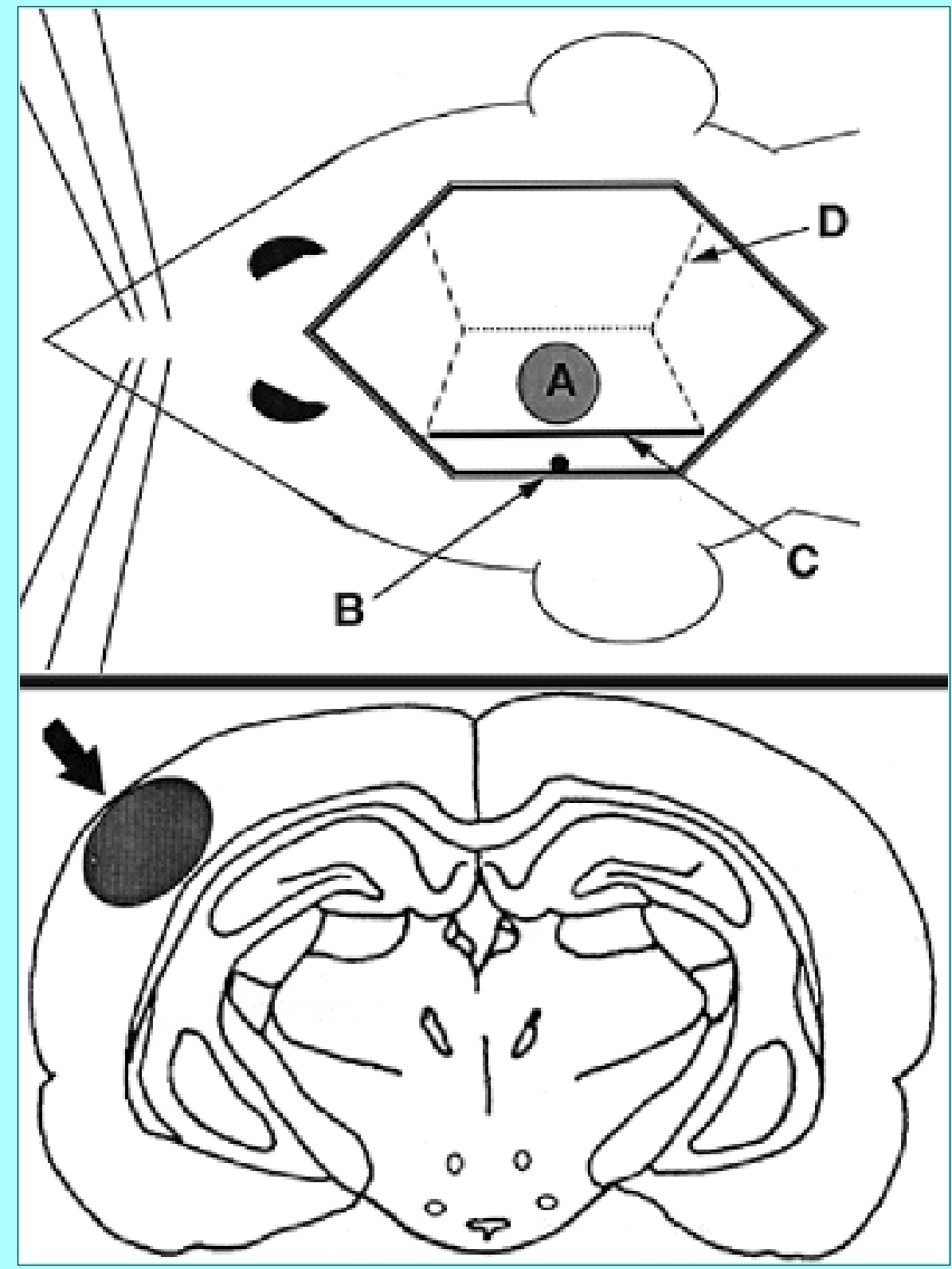

Fig. 1. Schematic illustrations depicting the operative site for transplantation. Upper: Craniectomy site for the attachment of the injury device for delivery of the fluid bolus (A). Burr-hole site for placement of transplant pipette and brain infusion cannula (B). Line marking the temporalis muscle insertion (C). Cranial sutures (D). Lower: Coronal schematic of the rat brain demonstrating the region of maximal cortical injury in the fluid-percussion brain injury model (shaded area) and the trajectory of insertion of the transplant pipette and 
infusion cannula.

The dura exposed via the burr hole was then incised with a 26-gauge needle. Simultaneously, a timed pregnant Sprague-Dawley rat (E16) was anesthetized with $60 \mathrm{mg} / \mathrm{kg}$ sodium pentobarbital injected intraperitoneally. An abdominal incision was made and a fetus was removed and placed in saline. The fetal brain was dissected and bilateral parietal cortical grafts $(1.5 \times 1.0 \times 0.5 \mathrm{~mm})$ were removed. These transplants were manually sliced into $0.2-\mathrm{mm}$ specimens and aspirated into a glass pipette that had an outer diameter of $0.9 \mathrm{~mm}$ to permit entry into the burr hole to a depth of $1.5 \mathrm{~mm}$. The aspirated specimen was then injected into the injured cortex over a period of 30 seconds. Approximately 200,000 to 300,000 cells were delivered using this technique. The total time from removal of the fetus to placement of the transplant was 2 to 4 minutes. The female rats were euthanized after harvesting of the fetal tissue. The brain-injured host animals were allowed to emerge from anesthesia while normothermia was maintained with a warming pad. Animals in Group 2 were prepared in a manner similar to Group 1 up to the point of transplantation. However, rather than fetal tissue, $50 \mu \mathrm{l}$ of saline was injected into the region of maximal cortical injury with identical protocols to those used for transplantation.

Animals in Group 3 were prepared identically to Group 1 animals up to, and including, the transplantation. Miniosmotic pumps (with an infusion rate of $0.5 \mu \mathrm{l} /$ hour) were filled with infusate 4 to 6 hours before starting the transplantation procedure. A brain infusion cannula was then attached to the pumps and the units were primed in sterile saline at $39^{\circ} \mathrm{C}$. Infusate included artificial cerebrospinal fluid (CSF), $0.1 \mathrm{mg} / \mathrm{ml}$ rat serum albumin, $25 \mu \mathrm{g} / \mathrm{ml} 7 \mathrm{~S} \mathrm{NGF}$, and $0.05 \mathrm{mg} / \mathrm{ml}$ gentamicin. The pumps were implanted subcutaneously in Group 3 animals. The brain infusion cannula was placed stereotactically to a depth of $1.5 \mathrm{~mm}$ in the cortex at the site of graft placement immediately after transplantation. The cannula was secured with dental cement and the skin was sutured over the apparatus. The pumps remained in place, providing a continuous infusion of NGF, until animals were euthanized at 72 hours, 1 week, or 2 weeks postinjury.

Group 4 animals underwent injury and preparation for transplantation identical to that for the previous groups. After harvesting the fetal tissue, the cells were placed in a suspension as previously described.[3,22] Briefly, after removal of cortical grafts from the fetus, the specimens were placed in iced $0.6 \% \mathrm{D}$-glucose in saline. The tissue was incubated at $37^{\circ} \mathrm{C}$ for 20 minutes in $10 \mathrm{ml}$ of the D-glucose solution and $2.5 \mathrm{mg}$ of trypsin. The transplant was then rinsed in D-glucose solution with DNase I $(1 \mathrm{mg} / 10 \mathrm{ml})$ and transferred to a final volume of $10 \mu \mathrm{l}$ of the same solution per fetal brain (approximately $1.5 \mathrm{~mm}^{3}$ cortical tissue per $10 \mu \mathrm{l}$ of volume). The cells were gently dissociated with a fire-polished Pasteur pipette. Cell viability was confirmed before and after transplantation with ethidium bromide exclusion. For transplantation, $7 \mu \mathrm{l}$ of the suspension were aspirated into a Hamilton syringe. This volume was chosen to deliver approximately the same volume of tissue (and cell number) used in Groups 1 and 3. The syringe was introduced into the injured cortex at the same coordinates used for Groups 1 to 3. The transplant was injected over a period of 5 minutes and the syringe was slowly removed. Animals in Group 4 underwent histological evaluation of transplant survival 2 weeks postinjury but did not undergo motor or cognitive evaluations. Procedures in all groups were performed under sterile conditions and normothermia was maintained by means of warming pads.

\section{Evaluation of Motor and Cognitive Function}

The evaluation of memory and motor function in rats undergoing a fluid-percussion brain injury has been described in detail.[29,45,46] All animals received preinjury training for cognitive testing in the Morris 
water maze, consisting of a circular tank $1 \mathrm{~m}$ in diameter filled with water maintained at $18^{\circ} \mathrm{C}$. The water surface was made opaque with a covering of styrofoam pieces. During training of the animals a submerged platform was present in the maze. Each rat underwent 20 training trials over a 2-day period prior to injury during which it learned to locate the platform using external visual cues. Immediately after completion of the last training trial, animals were anesthetized and injured as described above.

At 72 hours, 1 week, or 2 weeks postinjury, animals in Groups 1 to 3 were assessed for their ability to remember the learned cognitive task of locating the platform in the Morris water maze. For this evaluation the platform was removed from the maze and the animal's swimming pattern recorded with a computerized video system for 1 minute. The maze was separated into zones that were weighted according to their proximity to the platform. A memory score was generated by multiplying the weighted numbers by the time the animal spent in each zone and then adding these products.[18,34,45,46] Because an animal's cognitive function can only be tested once postinjury using this paradigm, animals underwent Morris water maze testing on the same day they were euthanized.

Animals in Groups 1 to 3 also underwent evaluation of neurological motor function at 72 hours, 1 week, or 2 weeks postinjury using previously described paradigms.[29,45] All testing was performed by an observer blinded to each animal's treatment. Animals were scored from 0 (severely impaired) to 4 (normal) for each of the following: 1) left and 2) right forelimb flexion during suspension by the tail; 3 ) left and 4) right hindlimb flexion when the forelimbs remain on a surface and the hindlimbs are lifted up and back by the tail; 5) the ability to resist lateral pulsion to the left and 6) right; and 7) the ability to stand on an inclined plane in the left, 8) right, and 9) vertical positions. For inclined plane scoring animals received a score of 4 by standing at a $45^{\circ}$ angle; 3 at $42.5^{\circ} ; 2$ at $40^{\circ}$; and 1 at $37.5^{\circ}$. A composite motor score (0-36) was generated by combining the scores for each of these tests.

Group 4 animals did not undergo motor or cognitive testing because there was no demonstration of transplant survival in animals receiving dissociated fetal cell grafts.

\section{Histological Evaluation}

Animals from Groups 1 to 3 were euthanized after cognitive testing at 72 hours ( 23 rats), 1 week (20 rats), 2 weeks (22 rats), or 3 weeks (six rats) postinjury, with an overdose of $200 \mathrm{mg} / \mathrm{kg}$ sodium pentobarbital administered intraperitoneally. Group 4 animals (eight animals) were all euthanized 2 weeks postinjury. Animals were perfused with intracardiac heparinized saline followed by $4 \%$ paraformaldehyde, then the brains were removed and stored in phosphate buffer until sectioned. All specimens were subsequently cut into $50-\mu \mathrm{m}$ sections on a vibratome, and Nissl-stained with toluidine blue to evaluate hippocampal cell loss and transplant survival.

Hippocampal CA3 damage was characterized in all animals at two identical regions (one rostral and one caudal hippocampal segment) by a blinded investigator. A score of 0 to 3 was derived for each region, with a score of 3 representing a normal-appearing CA3 region; a score of 2 representing a distinguishable region of cell loss due to a thinning of the pyramidal cell layer; a score of 1 representing a region in which continuity of the CA3 pyramidal cell layer was maintained by only a single cell in at least one portion; and a score of 0 representing a CA 3 region in which cell loss was severe enough to completely disrupt the continuity of the pyramidal cell layer in at least one segment. The scores from both the rostral and caudal sections were summed to provide a score of 0 to 6 for CA3 cell survival in each animal.

Two brains from animals in Groups 1 and 3, which had surviving transplants, also underwent a 
histological evaluation of transplant-host interaction using a modified Palmgren's silver stain.[15,35] Qualitative evaluation of 50- $\mu \mathrm{m}$ sections was performed using light microscopy.

\section{Statistical Analysis}

Memory, motor, and histological scores are ordinal measurements and therefore were evaluated using Kruskal-Wallis analysis of variance (ANOVA) followed by individual Mann-Whitney U tests. A probability value of less than 0.05 was considered statistically significant.

The procedures used throughout this study were approved by the institutional animal care and use committee of the University of Pennsylvania and were performed in accordance with standards published in the Guide for the Care and Use of Laboratory Animals, U.S. Department of Health and Human Services, Publication 85-23, 1985.

\section{RESULTS}

\section{Cognitive Outcome}

The rats subjected to fluid-percussion injury of moderate severity and treated with intraparenchymal injections of saline after injury (Group 2) demonstrated a significant cognitive deficit that did not differ when the animals were tested at 72 hours, 1 week, or 2 weeks. The stability of memory scores in the Morris water maze in animals undergoing fluid-percussion injury without pharmacological treatment has previously been documented between 48 hours and 2 weeks postinjury.[43,44] The scores of these brain-injured control animals were combined to create a median memory score of 67 for all injured control animals. Animals that received only fetal transplants (Group 1) demonstrated memory scores that were not significantly different from controls at 72 hours or 1 week postinjury (Fig. 2). However, by 2 weeks postinjury, brain-injured animals that received fetal transplants alone had memory scores (median of 78$)$ that were significantly better than vehicle-treated brain-injured animals $(p<0.05)$. 


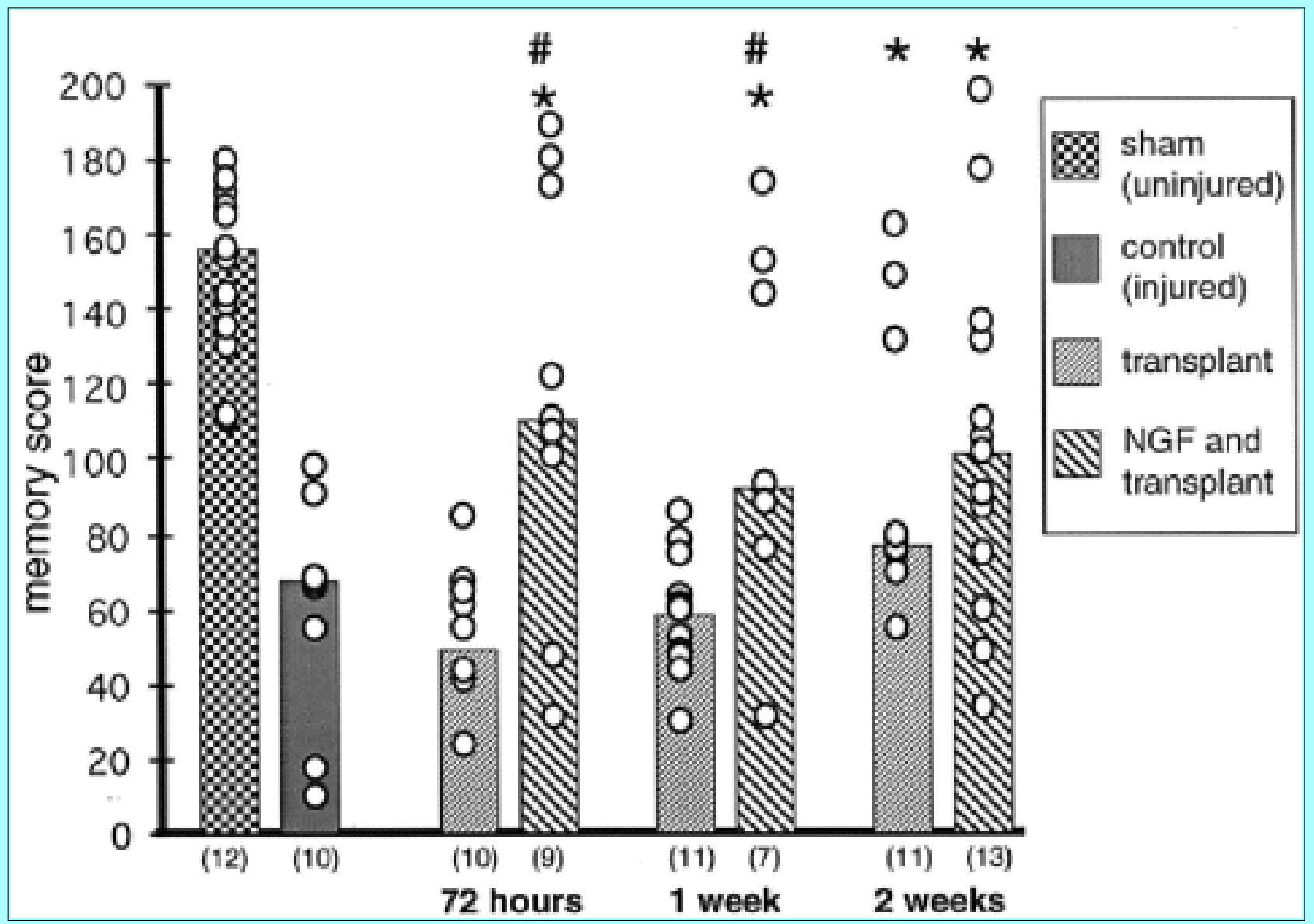

Fig. 2. Bar graph showing memory scores (open circles) of saline-treated, injured control animals compared to transplanted animals and transplant nerve growth factor (NGF)-treated animals at 72 hours, 1 week, and 2 weeks after fluid-percussion brain injury. The median memory score for the control animals was 67 . Asterisk indicates $p<0.05$ for transplant/NGF-treated animal's memory scores at all time points compared with control animals. Asterisk indicates $\mathrm{p}<0.05$ for transplant only animals' memory scores at 2 weeks compared with injured controls. Pound symbol indicates $\mathrm{p}<0.05$ for transplant/NGF-treated animals at 72 hours and 1 week compared with transplant only animals' memory scores at these same time points. For reference, uninjured (sham) animals from our laboratory, shown in the far left column, show a median memory score of 154 . Numbers of animals per group are shown in parentheses.

Brain-injured animals that received both NGF infusions and fetal cortical transplants differed significantly from injured control (saline-injected) animals and injured animals receiving transplant alone. At all three evaluation times the NGF/transplant animals had significantly improved memory scores (medians of 111, 93, and 102 at 72 hours, 1 week, and 2 weeks, respectively) when compared with controls (median of 67) ( $\mathrm{p}<0.05$ ). At 72 hours and 1 week postinjury the NGF/transplant animals also demonstrated significantly better memory scores (medians of 111 and 93) than animals receiving transplants alone (medians of 49 and 59) $(\mathrm{p}<0.05)$.

\section{Motor Outcome}

The results of transplantation with or without trophic factor infusion on postinjury neurological motor function are shown in Fig. 3. Injured control animals demonstrated a time-dependent improvement in motor scores from the 72-hour to the 1-week evaluation time and stabilized thereafter. Brain-injured 
animals receiving fetal transplants alone showed improved motor scores at all evaluation times when compared to injured controls. These differences, however, were statistically significant at only the 72-hour and 2-week evaluation times ( $\mathrm{p}<0.05)$. Animals receiving NGF treatment and fetal transplantation had significantly improved motor scores when compared to injured controls at all evaluation times, but these differences were also only significant at the 72-hour and 2-week times. At no time were the motor scores of transplanted animals and NGF/transplant animals significantly different.

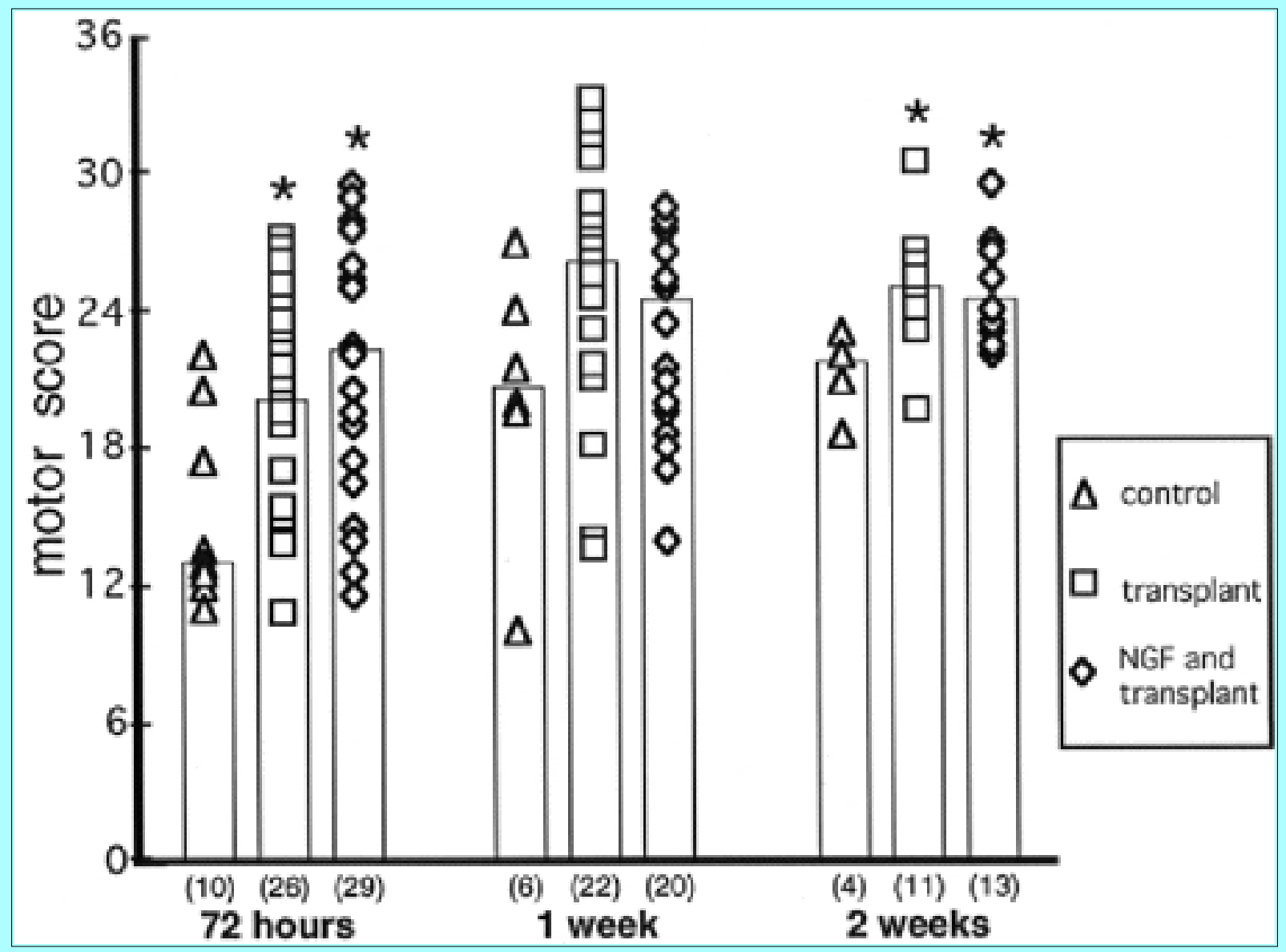

Fig. 3. Bar graph showing neurological motor scores of saline-treated, injured control animals, transplant only animals, and transplant/nerve growth factor (NGF)-treated animals at 72 hours, 1 week, and 2 weeks after injury. Asterisk indicates $p<0.05$ for transplant only and transplant/NGF-treated animals' motor scores at 72 hours and 2 weeks compared with injured control animals at the same time points. The differences between these groups at 1 week and between transplant only and transplant/NGF-treated animals at any time point were not significant. Numbers of animals per group are shown in parentheses.

\section{Histological Evaluation}

Detailed morphological evaluation revealed the characteristic formation of an injury cavity in the left parietal cortex and hippocampal cell loss in the CA3 region in all groups of rats. Scoring of hippocampal CA3 neuronal cell loss was performed in 16 transplanted animals, in 16 transplanted animals with NGF infusions, and in five control animals. Consistent loss of ipsilateral CA3 pyramidal cells was observed in all groups. The median score was 2.9 for transplanted animals, 3.1 for transplanted and NGF-treated animals, and 2.0 for control animals (probability was not significant).

Transplant viability was assessed at sacrifice for all evaluation times. There were no differences seen in 
the morphology of transplants related to the presence of NGF infusion. At 72 hours postinjury (48 hours posttransplantation) the transplants were best visualized as nests of cells interspersed in regions of host cortical necrosis (Fig. 4A and B). By 1 week postinjury these nests of transplanted cells were larger but remained quite discrete. By 2 weeks postinjury, the fetal transplants had grown quite large and, in many cases, completely filled the area of injury cavity formation (Fig. 4D and E). The normal gliotic lining of the injury cavity (Fig. 4C) was not apparent in such cases and the host and donor tissue could only be differentiated by cell morphology (Fig. 4E). Transplant survival was evident in 11 (68.8\%) of 16 animals receiving transplant alone and in $10(62.5 \%)$ of 16 animals also receiving NGF infusions $(\mathrm{p}=$ not significant). Group 4 animals, which received transplants of neuronal cell suspensions, demonstrated no transplant survival when evaluated 2 weeks posttransplantation (zero of eight animals).

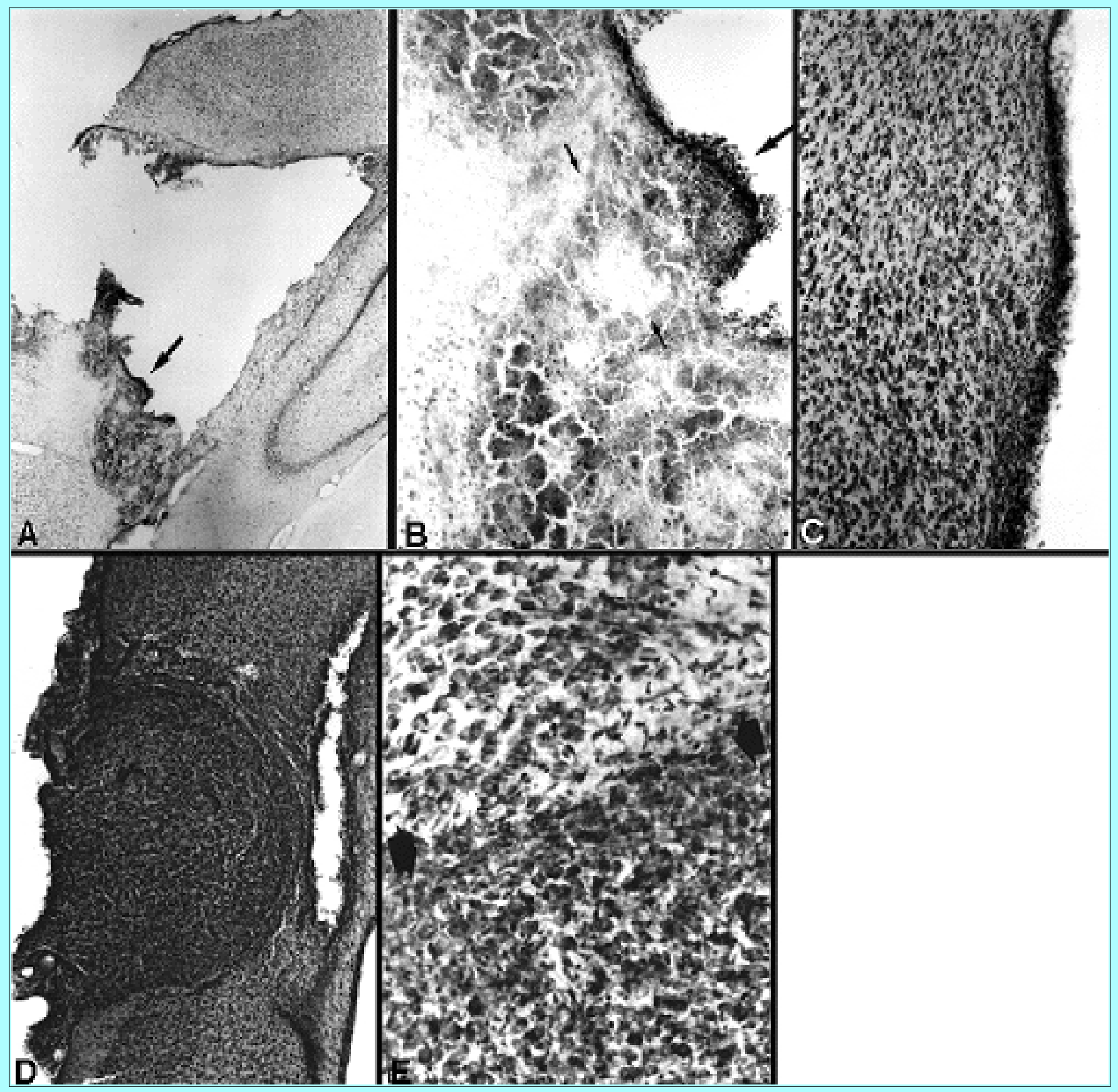

Fig. 4. Photomicrographs showing coronal $50-\mu \mathrm{m}$ sections of rat brain stained with toluidine blue. A: Injured, transplanted brain 72 hours postinjury. Much of the necrotic tissue has washed out during sectioning; the cortical injury cavity remains. The arrow identifies the 
region shown at higher magnification. Original magnification X 20. B: Higher power view demonstrating a nest of surviving transplanted cells (large arrow) inserted on the cavity wall adjacent to an area of necrosis and hemorrhage (small arrows). Original magnification $X$ 100. C: Injured, saline-treated control animal 2 weeks postinjury showing the layer of glial cells lining the cortical injury cavity. Original magnification X 100. D: Injured, transplanted brain 2 weeks postinjury demonstrating the filling of the region of the injury cavity with transplant. Original magnification X 40. E: Higher power view showing the host-graft interface (between the arrows). The host cortex is above the arrows and the graft below. Original magnification X 200.

Silver impregnation staining (modified Palmgren) revealed neural outgrowth both within the transplants and between the host and transplant. At 2 weeks postinjury the neuronal processes stained within the transplant; however, they appeared smaller and less dense than at the 3-week evaluation time (Fig. 5 left and center). The presence of neural processes crossing the interface between host and transplant was apparent by 3 weeks postinjury (Fig. 5 right).

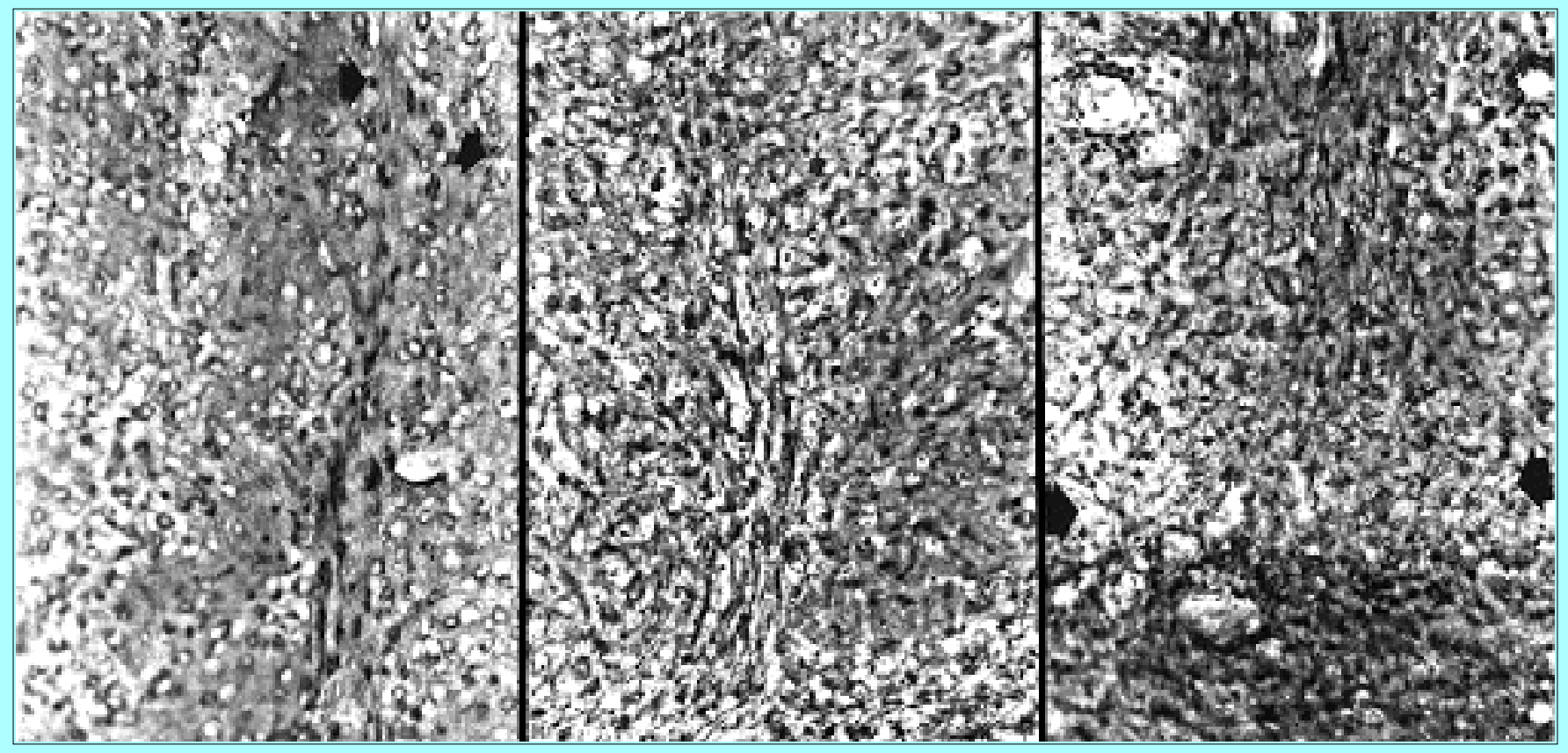

Fig. 5. Photomicrographs showing 50- $\mu$ m coronal sections of rat brain that had received transplants only postinjury. Left: Graft 2 weeks postinjury. Most cells do not have staining processes; however, there are occasional tracts of polar neurons with thin, staining processes (arrows). Center: Graft at 3 weeks postinjury demonstrates a higher percentage of staining processes and larger tracts. Right: Host-graft interface (between the arrows) 3 weeks postinjury. The host cortex is above the arrows and the graft below. There are staining neural processes crossing the interface. Modified Palmgren silver staining, original magnification X 200.

\section{DISCUSSION}

\section{Neural Transplantation to Improve Function}

Neural transplantation is a powerful tool to improve our understanding of neural development, plasticity, and responses to various types of injury. A more direct application involves the use of transplantation as a therapeutic intervention for diseases that have responded poorly to other treatments. By placing human 
fetal substantia nigra neurons in the caudate nucleus of patients with Parkinson's disease, investigators have noted initial clinical benefits. $[9,10,52]$ In contrast, models of spinal cord injury appear to require graft-host connectivity for functional improvements to occur. Fetal neural transplant survival, differentiation, and graft-host interactions have all been observed in acute and chronic models of spinal cord injury. $[23,37,38]$ After transplantation of fetal spinal cord tissue into the injury cavity produced by aspiration in the adult rat spinal cord, both afferents and efferents to and from the graft were identified between 6 and 56 weeks posttransplantation.[23] Anderson, et al.,[2] reported improvement in locomotor function in cats that received fetal neural transplants after a spinal cord injury produced by static-load compression. In two cats, a delayed deterioration in locomotor function correlated with active rejection of their grafts. Functional behavioral improvements have also been reported[21] after transplantation using a neonatal rat spinal cord injury model.

In a model of ischemic hippocampal injury, transplantation of fetal hippocampal cells has been shown to improve spatial memory.[31] Fetal grafts from various neural structures were placed in the damaged adult rat hippocampus. Improvements in water maze testing were noted only in those animals receiving grafts that included the fetal CA1 region. Attenuation of memory deficits has also been reported after transplantation of cholinergic cells into the hippocampus of rodents or primates following septohippocampal pathway lesions.[39,54]

\section{Neural Transplantation After Traumatic Brain Injury}

The extracellular environment of the injured cortex may be hostile to cellular viability during the first 24 hours postinjury.[16,47] Although transplants are more easily handled when they have been dissociated and placed in suspension, such treatment also exposes each cell to the pathological postinjury milieu. In the present study, the dissociation of fetal cells prior to transplantation resulted in uniform failure of all transplants.

In the absence of transplanted cells, the evolution of the fluid-percussion-induced injury cavity and glial scar, which is quite resistant to the passage of axonal processes, occurs unimpeded.[4,5,19,47] Previous studies have shown that transplantation of fetal cells 2 to 4 weeks postinjury, in this same model of brain injury, results in transplants that appear to be enclosed by the glia limitans and do not survive.[48,49] Studies demonstrating survival and appropriate migration of Purkinje cells transplanted into adult animals lacking normal Purkinje cells also suggest that a window may exist during which an organism may permit ingrowth of fibers or cells that are attempting to replace a missing cell type.[50,51] It is possible that the transplantation of fetal neural cells in our model at a somewhat later time (2-3 days postinjury) would still be within this hypothesized time window.

Initial studies using the fluid-percussion model of brain injury in rats have suggested that fetal transplants could attenuate hippocampal CA3 cell loss in the injured adult brain.[48] These observations raise the question of the ability of fetal transplants to improve cognitive deficits that normally occur after fluid-percussion brain injury. In the present study, we observed improvements in the cognitive deficits of brain-injured animals following transplantation of fetal cortical tissue only at 2 weeks postinjury. Because the cognitive deficits present after fluid-percussion brain injury appear to relate to hippocampal cell loss, $[18,44]$ transplants of hippocampal cells (or less-differentiated stem cells) rather than cortical cells may have resulted in further improvements.[7,20,26,31,39,49,54-56,58] Future studies will examine this hypothesis.

Neurological motor function in brain-injured animals receiving fetal transplants was significantly 
improved compared with control animals. To our knowledge, this is the first study to demonstrate such an effect in an experimental model of traumatic brain injury. Similar improvements observed in rodents who received transplants after undergoing frontal cortex aspiration lesions have been attributed to the presence of diffusible factors.[6]

The results of our histopathological analysis using silver staining suggest that transplanted cells in this model begin to differentiate and send processes across the boundary between transplant and host. Most prior work in cortical transplantation posttrauma has focused on aspiration or chemical-induced models of injury. These studies have elegantly shown anatomical and electrophysiological connectivity between host and transplant.[8,14,33,40,42] How these changes compare with transplant development in a more clinically relevant model of brain injury should be further evaluated.

\section{Role of Nerve Growth Factor}

Fetal neural transplants in cortical lesion models demonstrated survival and resulted in early improvements in neurological function. $[6,24,53]$ These improvements do not appear to be related to the presence of host/donor synaptic activity but rather to the production of soluble factors.[25] Although these benefits are not long-lasting, they provided evidence that manipulation of the neurotrophic environment could impact neural function after brain injury. Subsequent work using neurotrophic factor infusion paradigms has confirmed this hypothesis. $[17,43]$

Using the lateral fluid-percussion brain injury model, we have recently demonstrated improvements of memory scores with infusions of NGF alone.[43] Animals receiving only NGF infusions after brain injury did not show cognitive improvement until 1 week postinjury, compared to the improvement in memory scores observed in the present study at 72 hours postinjury in animals receiving fetal transplants in addition to NGF infusion. These data suggest that there may be synergistic effects between the fetal cell transplants and the neurotrophic factor infusion with respect to early improvements in cognitive behavior after fluid-percussion brain injury. No increased transplant survival or differences in axonal outgrowth in animals receiving NGF infusion were observed.

Evaluation of neurological motor tests was not different in injured animals with fetal transplants compared to injured animals receiving both fetal transplants and NGF administration. Whereas NGF has been shown to enhance survival of motor neurons in some circumstances, [57] these results suggest that the attenuation of motor dysfunction in animals receiving fetal transplants may be related to the release of other trophic factors, such as brain-derived neurotrophic factor, which may specifically interact with motor neurons.[12]

\section{CONCLUSIONS}

The present study demonstrates that fetal cortical neural cells can survive, begin to differentiate, and interact with the host brain when transplanted into a cortical lesion produced by means of the clinically relevant model of fluid-percussion brain injury in the rat. There are no detrimental behavioral effects from the transplant, and early synergistic effects may be triggered by adding NGF infusion. Further studies documenting the long-term histological and behavioral outcomes in this model are warranted because neural transplantation may offer the best chance for substantial functional recovery after traumatic brain injury.

\section{Acknowledgment}


The authors thank Dr. Eric L. Zager for his helpful review of the manuscript.

\section{References}

1. Aguayo AJ: Axonal regeneration from injured neurons in the adult mammalian central nervous system, in Cotman CW (ed): Synaptic Plasticity. New York: Guilford Press, 1985, pp 457-483

2. Anderson DK, Reier PJ, Wirth ED III, et al: Delayed grafting of fetal CNS tissue into chronic compression lesions of the adult cat spinal cord. Comp Neurol Neurosci 2:309-325, 1991

3. Björklund A, Stenevi U, Schmidt RH, et al: Intracerebral grafting of neuronal cell suspension I. Introduction and general methods of preparation. Acta Physiol Scand 522:1-7, 1983

4. Bovolenta P, Wandosell F, Nieto-Sampedro M: CNS glial scar tissue: a source of molecules which inhibit central neurite outgrowth. Prog Brain Res 94:367-379, 1992

5. Cortez SC, McIntosh TK, Noble LJ: Experimental fluid percussion brain injury: vascular disruption and neuronal and glial alterations. Brain Res 482:271-282, 1989

6. Dunnett SB, Ryan CN, Levin PD, et al: Functional consequences of embryonic neocortex transplanted to rats with prefrontal cortex lesions. Behav Neurosci 101:489-503, 1987

7. Dunnett SB, Whishaw IQ, Bunch ST, et al: Acetylcholine-rich neuronal grafts in the forebrain of rats: effects of environmental enrichment, neonatal noradrenaline depletion, host transplantation site and regional source of embryonic donor cells on graft size and acetylcholinesterase-positive fibre outgrowth. Brain Res 378:357-373, 1986

8. Fine A, Dunnett SB, Björklund A, et al: Transplantation of embryonic ventral forebrain neurons to the neocortex of rats with lesions of nucleus basalis magnocellularis--I. Biochemical and anatomical observations. Neuroscience 16:769-786, 1985

9. Freed CR, Breeze RE, Rosenberg NL, et al: Survival of implanted fetal dopamine cells and neurologic improvement 12 to 46 months after transplantation for Parkinson's disease. N Engl J Med 327:1549-1555, 1992

10. Freeman TB, Sanberg PR, Snow BJ, et al: The USF protocol for fetal nigral transplantation in Parkinson's disease. Neural Transpl 1:29, 1994 (Abstract)

11. Gennarelli TA: Animate models of human head injury. J Neurotrauma 11:357-368, 1994

12. Ghosh A, Carnahan J, Greenberg ME: Requirement for BDNF in activity-dependent survival of cortical neurons. Science 263:1618-1623, 1994

13. Giacobini MMJ, Hoffer BJ, Zerbe G, et al: Acidic and basic fibroblast growth factors augment growth of fetal brain tissue grafts. Exp Brain Res 86:73-81, 1991

14. Gonzalez MF, Sharp FR, Loken JE: Fetal frontal cortex transplanted to injured motor/sensory cortex of adult rats: reciprocal connections with host thalamus demonstrated with WGA-HRP. Exp Neurol 99:154-165, 1988

15. Goshgarian HG: A rapid silver impregnation for central and peripheral nerve fibers in paraffin and 
frozen sections. Exp Neurol 57:296-301, 1977

16. Hadani M, Freeman T, Munsiff A, et al: Fetal cortical cells survive in focal cerebral infarct after permanent occlusion of the middle cerebral artery in adult rats. J Neurotrauma 9:107-112, 1992

17. Hagg T, Manthorpe M, Vahlsing HL, et al: Delayed treatment with nerve growth factor reverses the apparent loss of cholinergic neurons after acute brain damage. Exp Neurol 101:303-312, 1988

18. Hicks RR, Smith DH, Lowenstein DH, et al: Mild experimental brain injury in the rat induces cognitive deficits associated with regional neuronal loss in the hippocampus. J Neurotrauma 10:405-414, 1993

19. Hicks RR, Smith DH, McIntosh TK: Temporal response and effects of excitatory amino acid antagonism on microtubule-associated protein 2 immunoreactivity following experimental brain injury in rats. Brain Res 678: 151-160, 1995

20. Hudson JL, Bickford P, Johansson M, et al: Target and neurotransmitter specificity of fetal central nervous system transplants: importance for functional reinnervation. J Neurosci 14:283-290, 1994

21. Iwashita Y, Kawaguchi S, Murata M: Restoration of function by replacement of spinal cord segments in the rat. Nature 367:167-170, 1994

22. Jacobs SE, Fine A, Juliano SL: Cholinergic basal forebrain transplants restore diminished metabolic activity in the somatosensory cortex of rats with acetylcholine depletion. J Neurosci 14:697-711, 1994

23. Jakeman LB, Reier PJ: Axonal projections between fetal spinal cord transplants and the adult rat spinal cord: a neuroanatomical tracing study of local interactions. J Comp Neurol 307:311-334, 1991

24. Kesslak JP, Brown L, Steichen C, et al: Adult and embryonic frontal cortex transplants after frontal cortex ablation enhance recovery on a reinforced alternation task. Exp Neurol 94:615-626, 1986

25. Kesslak JP, Nieto-Sampedro M, Globus J, et al: Transplants of purified astrocytes promote behavioral recovery after frontal cortex ablation. Exp Neurol 92:377-390, 1986

26. Kimble DP, Bremiller R, Stickrod G: Fetal brain implants improve maze performance in hippocampal-lesioned rats. Brain Res 363:358-363, 1986

27. Leonard JR, Maris DO, Grady MS: Fluid percussion injury causes loss of forebrain choline acetyltransferase and nerve growth factor receptor immunoreactive cells in the rat. J Neurotrauma 11:379-392, 1994

28. Madrazo I, Drucker-Colín R, Díaz V, et al: Open microsurgical autograft of adrenal medulla to the right caudate nucleus in two patients with Parkinson's disease. N Engl J Med 316:831-834, 1987

29. McIntosh TK, Vink R, Noble L, et al: Traumatic brain injury in the rat: characterization of a lateral fluid-percussion model. Neuroscience 28:233-244, 1989

30. Mouton PR, Olson L: Nerve growth factor increases the size of intracortical cholinergic transplants. Acta Neurol Scand 87:376-381, 1993

31. Netto CA, Hodges H, Sinden JD, et al: Foetal grafts from hippocampal regio superior alleviate ischaemic-induced behavioral deficits. Behav Brain Res 58:107-112, 1993 
32. Nieto-Sampedro M, Whittemore SR, Needels DL, et al: The survival of brain transplants is enhanced by extracts from injured brain. Proc Natl Acad Sci USA 81:6250-6254, 1984

33. Obukhova GP, Gogeliya KK, Senatorov VV, et al: Afferent and efferent connections of cortical transplants implanted into the damaged sensorimotor area of the cerebral cortex of adult rats. Neurosci Behav Physiol 22:1-6, 1992

34. Okiyama K, Smith DS, Thomas MJ, et al: Evaluation of a novel calcium channel blocker, (S)-emopamil, on regional cerebral edema and neurobehavioral function after experimental brain injury. J Neurosurg 77:607-615, 1992

35. Palmgren A: Specific silver staining of nerve fibers I. Technique for vertebrates. Acta Zoolog 41:239-265, 1960

36. Povlishock JT, Hayes RL, Michel ME, et al: Workshop on animal models of traumatic brain injury. J Neurotrauma 11:723-732, 1994

37. Reier PJ, Anderson DK, Thompson FJ, et al: Neural tissue transplantation and CNS trauma: anatomical and functional repair of the injured spinal cord. J Neurotrauma 9 (Suppl 1):S223-S248, 1992

38. Reier PJ, Stokes BT, Thompson FJ, et al: Fetal cell grafts into resection and contusion/compression injuries of the rat and cat spinal cord. Exp Neurol 115:177-188, 1992

39. Ridley RM, Thornley HD, Baker HF, et al: Cholinergic neural transplants into hippocampus restore learning ability in monkeys with fornix transections. Exp Brain Res 83:533-538, 1991

40. Rutherford A, Garcia-Munoz M, Dunnett SB, et al: Electrophysiological demonstration of host cortical inputs to striatal grafts. Neurosci Let 83:275-281, 1987

41. Santucci AC, Gluck R, Kanof PD, et al: Induction of memory and cortical cholinergic neurochemical recovery with combined fetal transplantation and GM1 treatments in rats with lesions of the NBM.

Dementia 4:273-281, 1993

42. Schulz MK, Hogan TP, Castro AJ: Connectivity of fetal neocortical block transplants in the excitotoxically ablated cortex of adult rats. Exp Brain Res 96:480-486, 1993

43. Sinson G, Voddi M, McIntosh TK: Nerve growth factor administration attenuates cognitive but not neurobehavioral motor dysfunction or hippocampal cell loss following fluid-percussion brain injury in rats. J Neurochem 65:2209-2216, 1995

44. Smith DH, Lowenstein DH, Gennarelli TA, et al: Persistent memory dysfunction is associated with bilateral hippocampal damage following experimental brain injury. Neurosci Let 168:151-154, 1994

45. Smith DH, Okiyama K, Thomas MJ, et al: Effects of the excitatory amino acid receptor antagonists kynurenate and indole-2-carboxylic acid on behavioral and neurochemical outcome following experimental brain injury. J Neurosci 13:5383-5392, 1993

46. Smith DH, Okiyama K, Thomas MJ, et al: Evaluation of memory dysfunction following experimental brain injury using the Morris water maze. J Neurotrauma 8:259-269, 1991 
47. Soares HD, Hicks RR, Smith DH, et al: Inflammatory leukocytic recruitment and diffuse neuronal degeneration are separate pathological processes resulting from traumatic brain injury. J Neurosci 15:8223-8233, 1995

48. Soares H, McIntosh TK: Fetal cortical transplants in adult rats subjected to experimental brain injury. J Neural Transplant Plast 2:207-220, 1991

49. Soares H, Sinson G, McIntosh TK: Fetal hippocampal transplants attenuate CA3 pyramidal cell death resulting from fluid percussion brain injury in the rat. J Neurotrauma (In press, 1995)

50. Sotelo C, Alvarado-Mallart RM: Embryonic and adult neurons interact to allow Purkinje cell replacement in mutant cerebellum. Nature 327:421-423, 1987

51. Sotelo C, Alvarado-Mallart RM, Frain M, et al: Molecular plasticity of adult Bergmann fibers is associated with radial migration of grafted Purkinje cells. J Neurosci 14:124-133, 1994

52. Spencer DD, Robbins RJ, Naftolin F, et al: Unilateral transplantation of human fetal mesencephalic tissue into the caudate nucleus of patients with Parkinson's disease. N Engl J Med 327:1541-1548, 1992

53. Stein DG, Palatucci C, Kahn D, et al: Temporal factors influence recovery of function after embryonic brain tissue transplants in adult rats with frontal cortex lesions. Behav Neurosci 102:260-267, 1988

54. Tarricone BJ, Simon JR, Low WC: Intrahippocampal transplants of septal cholinergic neurons: choline acetyltransferase activity, muscarinic receptor binding, and spatial memory function. Brain Res 632:41-47, 1993

55. Trojanowski JQ, Mantione JR, Lee JH, et al: Neurons derived from a human teratocarcinoma cell line establish molecular and structural polarity following transplantation into the rodent brain. Exp Neurol 122:283-294, 1993

56. Tuszynski MH, Buzsaki G, Gage FH: Nerve growth factor infusions combined with fetal hippocampal grafts enhance reconstruction of the lesioned septohippocampal projection. Neuroscience 36:33-44, 1990

57. Wayne DB, Heaton MB: The response of cultured trigeminal and spinal cord motorneurons to nerve growth factor. Dev Biol 138:473-483, 1990

58. Wictorin K, Brundin P, Gustavii B, et al: Reformation of long axon pathways in adult rat central nervous system by human forebrain neuroblasts. Nature 347:556-558, 1990

59. Wictorin K, Brundin P, Sauer H, et al: Long distance directed axonal growth from human dopaminergic mesencephalic neuroblasts implanted along the nigrostriatal pathway in 6-hydroxydopamine lesioned adult rats. J Comp Neurol 323:475-494, 1992

Manuscript received April 27, 1995.

Accepted in final form October 5, 1995.

This article was previously published in the Journal of Neurosurgery, Volume 84, pages 655-662. 
This study was supported, in part, by Grants NS26818 and NS08803 from the National Institute for Neurological Disorders and Stroke, National Institutes of Health, and by a Merit Review Grant from the Veterans Administration.

Address reprint requests to: Tracy K. McIntosh, Ph.D., Division of Neurosurgery, University of Pennsylvania School of Medicine, 3400 Spruce Street, Philadelphia, Pennsylvania 19104-4283. 